\title{
Reflets
}

Revue ontaroise d'intervention sociale et communautaire

\section{Dilemmes éthiques des soins aux personnes âgées}

\section{Noël Simard}

Volume 2, numéro 2, automne 1996

Vieillir à l'aube de l'an 2000

URI : https://id.erudit.org/iderudit/026129ar

DOI : https://doi.org/10.7202/026129ar

Aller au sommaire du numéro

Éditeur(s)

Reflets : Revue ontaroise d'intervention sociale et communautaire

ISSN

1203-4576 (imprimé)

1712-8498 (numérique)

Découvrir la revue

Citer cet article

Simard, N. (1996). Dilemmes éthiques des soins aux personnes âgées. Reflets, 2(2), 38-57. https://doi.org/10.7202/026129ar

Tous droits réservés (C) Reflets : Revue ontaroise d'intervention sociale et communautaire, 1996
Ce document est protégé par la loi sur le droit d'auteur. L'utilisation des services d'Érudit (y compris la reproduction) est assujettie à sa politique d'utilisation que vous pouvez consulter en ligne.

https://apropos.erudit.org/fr/usagers/politique-dutilisation/ 


\title{
Dilemmes éthiques des soins aux personnes âgées
}

\author{
Noël Simard \\ Professeur, Université de Sudbury
}

Parmi les problèmes éthiques posés par le vieillissement et le soin aux personnes âgées, nous trouvons, l'allocation des ressources, la compétence et le consentement éclairé. Dans la recherche d'une solution à ces problèmes, il faut d'abord réaffirmer la dignité personnelle de l'être humain, tout particulièrement de la personne âgée. Elle est aussi une personne et doit le devenir de plus en plus. La société doit se situer en fonction de la personne âgée et pour la personne âgée, en la reconnaissant et en la valorisant comme sujet autonome, actif et capable de prendre des décisions à propos de sa santé et de sa vie; la communauté doit toujours affirmer les droits de la personne âgée et favoriser son intégration et sa participation dans la société contre toute tentative d'automarginalisation et d'exclusion. Il faut aussi préciser les responsabilités : obligations de la société et des gouvernants dans la mise en place de programmes adéquats de soins de santé, rôle des institutions de soins médicaux, rôle des personnes âgées ellesmêmes et des membres de leur famille.

\section{Introduction}

Bien naitre, bien vivre, bien vieillir et, en fin de compte, bien mourir. Voilà un idéal que toute personne aimerait atteindre, 
"...que veut dire bien mourir ou vieillir en beauté?»
«...16\% de la population aura 65 ans et plus en l'an 2011...» mais qui présente bien des difficultés et des obstacles. Encore faut-il s'entendre sur le sens de ces expressions : que veut dire bien mourir ou vieillir en beauté? Tous les moyens sont-ils acceptables pour réaliser ces fins? Que faire lorsque les critères de qualité de vie entrent en conflit avec les critères touchant le respect de la vie? Comment agir avec les personnes qui ne peuvent plus exprimer leur opinion sur la manière dont elles aimeraient être traitées (ou ne pas être traitées)? Peut-on, ou doit-on considérer le facteur économique dans les soins à prodiguer pour maintenir les gens en bonne santé ou pour les aider à se rétablir? Ces questions touchent l'ensemble de la population de nos sociétés occidentales, mais elles se posent avec davantage d'acuité pour les personnes âgées.

En effet, les personnes âgées sont pour la plupart en bonne santé, mais plus que tout autre groupe d'âge, elles sont susceptibles de tomber malades, et par le fait même elles vont exiger davantage de soins médicaux. Avant d'aborder les problèmes éthiques des soins dispensés aux personnes âgées, il faut identifier et analyser les facteurs qui sont à l'origine de ces problèmes, du moins dans nos sociétés occidentales. Première constatation à faire, le nombre des gens âgés augmente rapidement. Au Canada, en 1921, l'espérance de vie était de 58,8 ans chez les hommes et de 60,6 ans chez les femmes. Or, les filles nées au Canada entre 1985 et 1987 peuvent espérer vivre près de 80 ans, alors que les garçons peuvent s'attendre à vivre 73 ans. En 1986, le groupe des personnes âgées de 65 ans et plus correspondait à $11 \%$ de la population. Si la tendance se poursuit, $16 \%$ de la population aura 65 ans et plus en l'an 2011, et cette proportion atteindra plus de $20 \%$ en l'an 2031 (Statistiques Canada 1992:69, 73)

Un second facteur important à considérer est celui de l'état de santé des gens âgés qui exige de plus en plus de soins. En effet, beaucoup d'entre eux ne peuvent se soigner eux-mêmes et ont besoin des services fournis par d'autres. Comme plus nous vieillissons et plus nous risquons de tomber malades, les progrès de la technologie médicale maintiennent souvent en vie les personnes âgées, mais dans un état d'incapacité. Il en résulte que le coût des soins médicaux pour ce groupe de patients est très élevé. En 
"Dans une situation économique difficile, le gouvernement peut-il offrir aux personnes âgées tous les services sociaux et de santé dont elles ont besoin?»
1981, les soins aux personnes âgées représentaient environ $40 \%$ des dépenses totales des services de santé au Canada (Canadian Medical Association $1987: 4$ )

Enfin, le nombre des membres de la famille qui pourraient prendre soin des gens âgés diminue, et le coût des solutions de rechange devient prohibitif. Diverses raisons expliquent ce phénomène: déclin radical du taux de natalité, d'où une baisse considérable du nombre des enfants qui pourraient à l'avenir prendre soin de leurs parents âgés, augmentation des divorces, des séparations et des remariages chez les adultes jeunes ou d'âge moyen, ce qui contribue à mettre les personnes âgées à l'écart, augmentation des institutions ou foyers pour vieillards, nombre croissant de personnes âgées célibataires ou de veuves qui n'ont personne pour prendre soin d'elles, etc.

Ces faits soulèvent bien des questions sur la manière de répondre aux besoins des personnes âgées quand il s'agit des services de santé et des personnes qui vont s'en charger. Ainsi, les enfants ont-ils l'obligation de s'occuper de leurs parents âgés et de leur assurer des soins adéquats? Ont-ils une part à payer s'ils ne peuvent assumer eux-mêmes ces services? Quelles sont les obligations de la société?

Dans une situation économique difficile, le gouvernement peut-il offrir aux personnes âgées tous les services sociaux et de santé dont elles ont besoin? Ce sont là des dilemmes éthiques, puisqu'ils nous obligent à comparer les droits et les responsabilités des divers individus et groupes de la société.

Dans les pages qui vont suivre, nous allons d'abord identifier et analyser les problèmes éthiques majeurs posés par le vieillissement et le soin aux personnes âgées. En cherchant une solution à ces problèmes, si solution il $\mathrm{y}$ a, nous rappellerons les principes fondamentaux dont il faut absolument tenir compte, et nous préciserons les responsabilités des personnes concernées. 


\section{Problèmes d'éthique}

«Il n'est pas étonnant que les dilemmes éthiques posés par le soin aux personnes âgées soient nombreux, car les principales sources de perplexité morale se relient aux questions sur la fin de la vie et la compétence des malades.»
Le mot «problème» ou dilemme est peut-être celui qui est le plus fréquemment employé dans les textes de bioéthique. De fait, celle-ci, jusqu'à maintenant, s'est surtout employée à analyser et à résoudre des problèmes. Mais qu'est-ce qu'un problème d'éthique? En général, on peut le définir comme étant une question pour laquelle les réponses jusqu'ici proposées sont incertaines ou contradictoires. D'ordinaire, un problème implique un conflit ou une incertitude à propos de la ligne de conduite à adopter ou à maintenir. Ces conflits peuvent provenir de différences de perspective, d'horizon, de perception, d'hypothèse et de valeur. En effet, les conflits surgissent parce qu'il y a un manque de preuves et d'expériences ou parce qu'on n'a que des perceptions partielles de la réalité, ou encore parce qu'il y a concentration sur une valeur ou divergence au niveau de l'éthos, de la moralité ou de l'éthique.

Les dilemmes éthiques ou conflits entre différents principes fondamentaux obligent alors le professionnel de la santé à privilégier au moins l'un des principes au détriment des autres, ce qui peut se traduire par une certaine "perplexité morale», c'est-àdire le choix de ce qui doit être fait dans une situation précise, tout bien considéré (Harvey 1993 : 54).

L'incertitude ou l'ambiguïté en ce qui concerne la ligne de conduite à adopter peut être d'ordre factuel (l'administration de tel analgésique en telle quantité accélère-t-elle la mort?), d'ordre éthique ( $\mathrm{y}-\mathrm{a}-\mathrm{t}-\mathrm{il}$ une différence entre ne pas initier un traitement et le cesser, entre laisser mourir et faire mourir?), ou bien d'ordre juridique (l'aide au suicide est-il un crime?). Il n'est pas étonnant que les dilemmes éthiques posés par le soin aux personnes âgées soient nombreux, car les principales sources de perplexité morale se relient aux questions sur la fin de la vie et la compétence des malades. Parmi ces problèmes, nous analyserons l'âgisme, l'allocation des ressources, la compétence et le consentement éclairé. 


\section{L'âgisme ou le manque de respect pour les personnes âgées}

"Considérées comme des «victimes» faciles, les personnes âgées sont souvent l'objet de mauvais traitements physiques et mentaux de la part des membres de leur famille ou $d u$ personnel des maisons de retraite.»
Sans être un dilemme proprement dit, l'âgisme est tellement présent dans notre société qu'il est important d'y consacrer quelques lignes.

Il est généralement admis que la discrimination fondée sur la race (racisme) ou sur le sexe (sexisme) est contraire à la dignité de la personne et ne devrait pas être tolérée. Au cours des dernières décennies, l'âgisme est venu s'ajouter au racisme et au sexisme dans la liste des attitudes qui violent les normes courantes des droits de la personne. Nous pouvons décrire l'âgisme comme la ségrégation à l'encontre des personnes du fait de leur âge. Ses manifestations vont varier d'un pays à l'autre. Au Canada, par exemple, on le trouve dans les lois et les normes sur la retraite obligatoire à 65 ans, dans le paternalisme des soins médicaux et infirmiers, ou encore dans les formes extrêmes de mauvais traitements physiques ou mentaux infligés aux gens âgés. Expression plus insidieuse de l'âgisme, le paternalisme est fort répandu chez les professionnels de la santé. Il s'exprime par une attitude condescendante et infantilisante, en considérant l'âge avancé comme une maladie débilitante qui place les personnes âgées au même niveau que de petits enfants ou d'incompétents mentaux, incapables de prendre des décisions sensées en ce qui concerne leur santé ou leur bien-être. Considérées comme des «victimes» faciles, les personnes âgées sont souvent l'objet de mauvais traitements physiques et mentaux de la part des membres de leur famille ou du personnel des maisons de retraite. Cette violence à leur endroit est reconnue comme un problème social qui va s'aggravant, et dont on commence à peine à mesurer l'étendue.

Face à ces manifestations ou expressions d'âgisme, il faut promouvoir et développer le respect des personnes âgées dont la vie, si réduite soit-elle, doit être protégée, défendue et valorisée au plus haut point. 


\section{Allocation des ressources}

L'allocation des ressources représente un autre dilemme éthique important. On constate dans les pays d'Amérique du Nord et d'Europe un vieillissement de la population. En effet, la population âgée s'accroît tant en nombre absolu qu'en pourcentage. Par conséquent, les besoins en soins de santé exigent de plus en plus de ressources et de services. Les changements démographiques, les progrès technologiques dans le domaine de la santé, de même que l'augmentation constante des coûts de santé, expliquent pourquoi la répartition des ressources est devenue une question débattue en public. Certains se demandent si les personnes âgées ne reçoivent pas plus que leur juste part de ces ressources. Les opinions sur ce problème varient depuis le rejet de l'âge avancé comme critère pour le traitement (Daniels 1987) jusqu'à l'encouragement au suicide et à l'euthanasie des personnes âgées avant qu'elles n'aient besoin de soins coûteux (Bottin 1987). Entre ces positions extrêmes, nous trouvons des opinions intermédiaires qui voient dans l'âge avancé un facteur plus ou moins important pour décider du traitement médical approprié. Dans cette optique, Callahan (1987) distingue l'âge en tant que critère médical ou technique, et l'âge en tant que critère centré sur une personne

"...l'âge ne doit pas

être un critère médical, car ce n'est pas en soi un motif légitime pour s'abstenir d'un traitement ou pour l'interrompre.» ou un patient ${ }^{1}$. Selon lui, l'âge ne doit pas être un critère médical, car ce n'est pas en soi un motif légitime pour s'abstenir d'un traitement ou pour l'interrompre. En effet, certaines personnes octogénaires peuvent recouvrer la santé grâce à une opération chirurgicale qui ne serait d'aucun secours à un sexagénaire. Par contre, toujours selon Callahan, l'âge peut et doit être considéré comme un facteur parmi d'autres dans la décision de traiter ou de ne pas traiter. La médecine devrait renoncer à l'acharnement thérapeutique, c'est-à-dire au traitement à tout prix, pour se consacrer davantage à soulager la souffrance des personnes âgées et à améliorer la qualité physique et mentale de leur vie. Quoi qu'il en soit, la question se pose clairement: peut-on utiliser l'âge comme critère pour limiter l'accès à certains biens et services de santé? Les différences d'âge peuvent-elles être considérées comme critère acceptable de répartition de biens ou de services rares? 
Ou encore peut-on justifier le rationnement des ressources en se basant sur l'âge?

\section{Compétence et consentement éclairé}

Les questions liées à la compétence des malades âgés soulèvent beaucoup de préoccupations tant chez les professionnels des soins de santé que chez les malades. En termes simples, la compétence est «la capacité de s'acquitter d'une tâche», et les évaluations de compétence sont «des procédures médico-légales conçues pour évaluer la capacité d'un sujet de comprendre les conséquences de ses décisions et de ses gestes et de fonctionner normalement» (Harvey 1993: 56) ${ }^{2}$. La compétence de la personne âgée devient un élément clef dans les décisions de traitement. En règle générale, les personnes âgées ont le même droit moral et légal de prendre des décisions sur leurs soins de santé que tout autre individu autonome. Cependant, elles sont exposées à des problèmes médicaux qui peuvent diminuer leur capacité de prendre des décisions. Par conséquent, dans leur cas, la doctrine du consentement éclairé exige une certaine adaptation.

Ainsi, les personnes âgées compétentes ont le droit de refuser certains traitements, comme les traitements de prolongation de vie comme la réanimation cardio-pulmonaire, l'alimentation et l'hydratation par moyens artificiels, le contrôle de l'infection par des antibiotiques, pour la raison qu'elles jugent ces traitements contraignants. Cependant, elles doivent avoir accès à tous les

"Une personne âgée qui refuse un

traitement qui lui sauverait la vie est-elle plus incompétente que celle qui refuse de cesser de boire ou de fumer...» services appropriés et nécessaires. Les malades âgés compétents peuvent déterminer ce qui, à leurs yeux, constitue des avantages ou des préjudices, et les risques qui valent la peine d'être pris. Cela provoque souvent des inquiétudes chez les professionnels de la santé : faut-il forcer à manger un vieillard qui refuse de le faire par manque de goût de vivre ou par désir de mourir? Une personne âgée qui refuse un traitement qui lui sauverait la vie est-elle plus incompétente que celle qui refuse de cesser de boire ou de fumer, ou qui traverse la rue au feu rouge? 
Le traitement des personnes âgées incompétentes pose un problème encore plus délicat. Dans le cas d'incapacité totale et permanente, causée par exemple par une démence profonde, selon quels critères les préposés aux soins devront-ils décider du traitement approprié: les désirs que le patient a exprimés alors qu'il était autonome, les meilleurs intérêts du patient, les directives d'autres personnes «importantes» comme les membres de la famille ou les amis?

Le Collège royal des médecins et chirurgiens du Canada recommande à ses membres d'adopter la démarche suivante :

Lorsqu'on doute de la capacité du patient à décider, le médecin doit s'efforcer de pallier cette incertitude en recourant à la consultation médicale, avant d'obtenir le consentement éclairé. Si les décisions du patient ou son refus de prendre une décision semblent déraisonnables et susceptibles de lui causer de graves dommages, le médecin doit consulter les membres de la famille, ses collègues et les autorités de l'établissement hospitalier afin de déterminer s'il faut agir à l'encontre des voeux exprimés par le patient, ou de décider pour lui en l'absence de voux clairement exprimés, tout au moins jusqu'à ce que la capacité du patient ne fasse plus de doute (Royal College of Physician and Surgeons of Canada 1988:21/2 (encarté))

Les autres personnes préposées aux soins, tout comme les travailleurs sociaux et les membres de la famille, doivent aussi respecter les décisions de la personne âgée, si déraisonnables qu'elles puissent paraitre, en autant qu'elles ne causent pas un dommage grave. La capacité du malade étant requise pour un consentement informé, ce qui constitue un droit fondamental (Harvey 1993) ${ }^{3}$, il s'ensuit que les professionnels de la santé et les membres de famille doivent chercher à protéger l'autonomie dès qu'elle est menacée. Dans la mesure du possible, ils doivent éviter d'administrer des médicaments susceptibles de réduire la capacité de décision chez la personne âgée. Du fait que l'incapacité peut frapper à tout moment, n'est-il pas souhaitable et recommandé 
que les gens âgés rédigent à l'avance des directives qui garantissent que leurs volontés seront connues des préposés aux soins et des mandataires? Enfin, afin de passer outre à ses décisions, il est contraire à l'éthique de considérer la personne âgée comme inapte.

\section{Principes et valeurs}

Dans la recherche d'une solution à ces problèmes, si solution il y a, il faut d'abord se référer à des valeurs et à des principes fondamentaux. Nous en analyserons quelques-uns, à savoir la dignité de la personne âgée, l'éthique des soins personnels et la justice.

\section{Dignité de la personne âgée}

Toute réflexion au sujet de l'éthique et des décisions concernant les personnes âgées doit s'appuyer sur un principe fondamental largement reconnu dans la société contemporaine, celui de la dignité personnelle de l'être humain. De ce principe nous pouvons déduire une triple affirmation essentielle:

a) la personne âgée, elle aussi, est une personne,

b) elle doit le devenir de plus en plus,

c) la communauté doit se situer en fonction de la personne âgée.

«Dans une société qui privilégie les valeurs de l'avoir, du pourvoir et du plaisir, il est pertinent de réaffirmer que la personne âgée elle aussi est une personne.»
Dans une société qui privilégie les valeurs de l'avoir, du pourvoir et du plaisir, il est pertinent de réaffirmer que la personne âgée aussi est une personne. Comme de nombreuses personnes âgées n'entrent pas dans ces catégories de l'avoir, du pouvoir et du plaisir, leur sort est souvent la marginalisation. Pour illustrer ce phénomène, nous n'avons qu'à penser aux décisions sociales qui contraignent la personne âgée à s'éclipser, à se réfugier dans sa vie privée, à ne causer aucun problème aux autres. Nous n'avons qu'à faire remarquer cette conjuration du silence qui pèse sur les vieillards et sur leurs problèmes, et la déconcertante superficialité avec laquelle sont rendus publics les remèdes présumés aptes à éliminer la marginalisation (Burgalassi 1977). 
"La personne

âgée... a le devoir de

grandir dans sa dignité personnelle, en épanouissant son être propre au contact des autres et pour les autres.»
Ayant surtout comme fondement de l'existence la «trilogie» production-consommation-profit, notre société et notre culture ont tendance à évaluer les personnes non en fonction de leur être, mais de leur avoir et de leur faire. Il est alors clair que la personne âgée ne pourra figurer dans cette société qu'à titre marginale. Dans un tel contexte, il est nécessaire de remonter aux sources de la personnalité pour y retrouver ce qui est commun à tous: l'être dans toute sa dignité et son égalité, la nature humaine propre à chaque stade de l'existence de la personne, du premier instant jusqu'au dernier. La dignité de l'être humain est à la fois un attribut propre à la personne, de toujours et de toute condition, et une responsabilité remise entre ses mains. De là découle l'autodétermination de la personne, la prise en main de sa vie.

La personne âgée est une personne et doit de plus en plus devenir une personne, c'est-à-dire qu'elle doit, elle aussi, réaliser progressivement les attributs de la personne (subsistance, ouverture à l'infini, réalisation de soi, relation, incarnation, dimension sociale, liberté, etc.) qui sont en étroite relation avec les valeurs et les exigences précises de communion et de don (Simard 1984). La personne âgée se doit d'être comme toute personne un être "avec» et "pour» les autres. Elle a le devoir de grandir dans sa dignité personnelle, en épanouissant son être propre au contact des autres et pour les autres. Elle doit refuser de céder à la tentation d'automarginalisation, et travailler plutôt à son insertion et à sa participation à la vie de la communauté et de la société. En effet, la personne âgée doit lutter contre les tendances injustes de la culture moderne qui la marginalisent, la contraignent à l'isolement et l'amènent à renoncer à son rôle et à son statut social; il lui faut aussi combattre la tendance à renoncer à sa propre place dans la société, ce qui s'enracine le plus souvent dans la conviction, chez les gens âgés, qu'il sont inutiles et qu'ils constituent une charge pour la société.

Enfin, la personne âgée doit devenir un sujet actif et responsable dans la société par la redécouverte et la valorisation de ses dons et talents propres (expérience de vie, sagesse, etc.). Par ailleurs, la société doit aider la personne âgée à grandir en lui offrant les moyens indispensables à sa croissance et à sa participation. De 
"La personne âgée doit demeurer un sujet actif, et non l'objet ou le simple destinataire d'un certain nombre de services..." façon négative, la société a le devoir de combattre les diverses formes de marginalisation. De façon positive, elle a le devoir de valoriser la personne âgée en lui facilitant la jouissance de ses droits et l'accomplissement de ses devoirs. Bien entendu, la défense et la promotion des droits et devoirs de la personne âgée ne peuvent se faire qu'avec et par elle. La personne âgée doit demeurer un sujet actif, et non l'objet ou le simple destinataire d'un certain nombre de services et de prestations sociales.

Avant d'être des vieillards, les gens âgés sont des personnes. D'ailleurs l'expression "personne âgée» dénote cette préoccupation éthique du respect de la dignité. Dans la mesure où une personne est traitée comme telle, l'acceptation de sa vieillesse devient chose facile. Le respect de cette dignité ou la reconnaissance de cette qualité de personne présuppose que la personne a le droit de faire ses propres choix selon les valeurs qu'elle privilégie, d'après sa perception personnelle de la situation, et selon les contraintes environnementales et sociales ou pressions culturelles qu'elle aussi subit (Kenyon-Davidson 1993).

\section{Éthique de soins personnels}

Les patients âgés ont le droit, non pas seulement l'intérêt ou la préférence, d'être traités par des médecins et des infirmières selon une éthique de soins personnels, c'est-à-dire selon une relation entre le professionnel de la santé et le malade qui soit réellement humaine. Or, cette relation est authentiquement humaine si elle manifeste quatre caractéristiques fondamentales: humanité, lucidité, autonomie et fidélité (Fried 1974). La caractéristique de l'humanité souligne le fait que chaque personne est un individu distinct avec une biologie par conséquent distincte, ainsi que des besoins, faiblesses, points forts et un plan de vie individualisé.

"C'est surtout le principe d'autonomie qui fonde la norme $d u$ consentement informé et éclairé.» L'autonomie implique le besoin et la capacité de décider de ses buts personnels et la liberté d'agir en conséquence. C'est surtout le principe d'autonomie qui fonde la norme du consentement informé et éclairé. Ce dernier protège les gens âgés contre les intrusions inacceptables des médecins et infirmières dans leur corps et leur vie. Il permet à la personne âgée de garder le contrôle de 
«... la relation entre

les professionnels de la santé et les malades doit remplir les critères de confiance et de fidélité.» sa vie et favorise un dialogue véritable pour permettre un réel partage de la prise de décision.

Pour obtenir le consentement de la personne âgée, il faut lui fournir, comme à tout autre malade, des renseignements adéquats sur la nature, la durée, les risques et les avantages du traitement, sur les conséquences prévisibles et les alternatives. Cette information doit être fournie en termes compréhensibles pour la personne âgée. En outre, celle-ci doit avoir l'occasion d'y réfléchir et d'en parler au préalable avec les membres de sa famille ou à d'autres personnes.

Le caractère «informé» du consentement représente une autre caractéristique de la relation entre les professionnels de la santé et les malades, à savoir la lucidité. Cela signifie une communication franche, prête à dispenser tout renseignement connu qui puisse servir à l'auto-détermination, à la prise de décision et au choix d'alternatives dans la réalisation de ses projets. La lucidité est mal servie si les professionnels de la santé perçoivent l'obtention du consentement comme un mot de passe légal dont ils ont besoin pour faire leur besogne. Ils ne comprennent pas alors que les renseignements adéquats sont pour le patient une nécessité primordiale, ainsi qu'une exigence morale de l'intégrité de la relation. Le consentement n'est pas seulement une sorte de rite imposé par la loi. Il faut l'interpréter comme un acquiescement et un accord. Cela suppose une liberté d'agir qui se reconnaît à l'absence de contrainte et de manipulation. Et en cas d'inaptitude, provenant d'une déficience physique ou mentale qui rend impossible l'exercice de la capacité décisionnelle, Saint-Arnaud, (1993), il faut rechercher le consentement implicite, apparent ou présumé, c'est-à-dire essayer de connaître les désirs du patient, soit en se référant aux directives données à l'avance ou au testament de vie.

Enfin, pour qu'elle soit authentiquement humaine, la relation entre les professionnels de la santé et les malades doit remplir les critères de confiance et de fidélité. La fidélité signifie que l'on répond loyalement aux attentes justifiées des patients. Quand ces derniers entrent en relation avec les médecins, ils s'attendent à bon droit, bien qu'implicitement, à ce que ces derniers soient 
dûment qualifiés, c'est-à-dire à la hauteur des normes actuelles de la médecine. La relation entre les professionnels de la santé et les malades doit donc comporter non seulement la présence personnelle du professionnel et le souci du patient, mais aussi la compétence et les services spécialisés. Cela rejoint les principes de bienfaisance et de bienveillance du serment d'Hippocrate. Non seulement le médecin ne doit pas nuire à son patient mais lui faire du bien, il doit aussi lui vouloir positivement du bien. Un engagement personnel et professionnel est nécessaire pour faire face avec compétence aux réalités de la maladie, de la douleur, de la souffrance, de la perte d'autonomie et de la mort, et, en cas d'échec, pour entourer d'affection les personnes affligées. Chez le médecin, cet engagement signifie la disposition à confronter sa propre vulnérabilité face à la maladie incurable, la souffrance et la mort imminente. Il s'agit alors de ne pas abandonner les patients, surtout lorsque la compétence professionnelle et la médecine ont atteint leurs limites.

Au cours des dernières décennies, bien des incertitudes, des conflits au chevet des mourants et des causes devant les tribunaux ont montré qu'il n'est pas toujours facile pour le médecin de satisfaire à l'exigence de fidélité. Très souvent, il se trouve dans des situations où l'équilibre entre deux devoirs fondamentaux (comme celui de sauver la vie et celui d'empêcher la souffrance) est difficile à établir. Jusqu'où un médecin doit-il respecter le refus d'un traitement vital pour un patient âgé dont l'autonomie est douteuse?

\section{Principe de justice}

Le soin des personnes âgées et l'allocation des ressources nous obligent à faire appel à un autre principe fondamental, celui de la justice ou de l'équité dans la répartition des avantages et des problèmes dans la société. En effet, lorsqu'une société étudie la façon de distribuer ses ressources, elle doit tendre à un équilibre entre l'idéal de liberté ou d'autonomie et celui d'égalité. Même quand le droit aux soins de santé est reconnu, la santé ne représente 
qu'un bien parmi d'autres. Et lorsque la quantité de ressources disponibles est limitée, la question de justice distributive se pose. Encore faut-il s'entendre sur cette notion de justice. C'est là un défi de taille, car au sein de toutes les activités qui sont réalisées pour procurer aux individus et aux groupes défavorisés, le maximum de justice, il y a beaucoup de confusion et de désaccord sur ce que signifie la justice; il n'existe aucun principe reconnu de justice qui permettrait à une seule personne d'avoir accès à tous les traitements ou services avantageux pour elle.

Dans cet ordre d'idées, Kathleen Cranley Glass (1993) rappelle que diverses approches théoriques sont possibles en matière de la répartition des ressources sociétales: contribution envers la société, aptitude, mérite ou réalisation, effort, besoin et égalité. Certaines approches comme la contribution envers la société ou la valeur sociale, l'aptitude, le mérite ou les réalisations, ou encore l'effort, posent de sérieux problèmes lorsqu'on tente de les appliquer au rationnement des services et soins de santé. La valeur sociale, qui accorde la primauté à des facteurs comme l'importance, le rang, ou la productivité dans la société, est nettement arbitraire et discriminatoire. En effet, ce critère désavantage les personnes âgées lorsqu'on considère les contributions actuelles et non les contributions passées, ce qui risque d'exclure très facilement les personnes handicapées physiques ou mentales qui, en apparence, sont peu rentables et productives sur le plan social. Ce critère va donc à l'encontre de la dignité de la personne qui mérite respect du seul fait de la condition humaine, et non en qualité d'instrument utile à la société.

Rationner les ressources en se basant sur l'aptitude, le mérite ou les réalisations, c'est comparer la conduite des gens et évaluer

"Les soins de santé doivent être fournis à partir de besoins en matière de prévention ou en matière de traitement, et non être attribués au mérite.» leur rendement et leur contribution. Les soins de santé doivent être fournis à partir de besoins en matière de prévention ou en matière de traitement, et non être attribués au mérite. Comme le note Cranley Glass, "toute distinction entre les patients qui «méritent» d'être malades (ils sont donc moins dignes de profiter des ressources de santé) et ceux qui ne le méritent pas constitue la discrimination la plus odieuse» (1993:78). De même, la répartition fondée sur l'effort est arbitraire et contraire à l'équité, car 
"...l'égalité de tous les individus ne signifie pas qu'ils auront droit à des traitements égaux ou identiques, mais bien que tous les patients qui ont besoin de tel ou de tel traitement seront considérés sur un pied d'égalité.» l'inégalité des revenus n'est pas nécessairement attribuable à l'inégalité de l'effort.

Si l'on privilégie l'approche des besoins, certains autres problèmes surgissent. Par exemple, les personnes âgées ont généralement des besoins plus importants en matière de santé. Cela veut-il dire que tous leurs besoins doivent être satisfaits? Lorsque les ressources sont insuffisantes pour répondre à tous les besoins, il faut que ceux-ci soient étroitement définis d'après la limitation des ressources, ou alors que le critère de rationnement soit associé à d'autres éléments comme le principe d'égalité. Comme l'explique encore Cranley Glass, «la répartition fondée sur l'égalité de tous les individus ne signifie pas qu'ils auront droit à des traitements égaux ou identiques, mais bien que tous les patients qui ont besoin de tel ou de tel traitement seront considérés sur un pied d'égalité» (1993:80). Le critère de l'égalité exclut très clairement la répartition basée sur la valeur sociale ou sur l'effort, en éliminant toute possibilité de discrimination basée sur la race, la religion, le sexe ou l'âge, à moins que ces caractéristiques ne soient pertinentes pour le traitement médical. Par conséquent, la répartition des ressources basée uniquement sur l'âge est à rejeter; elle équivaut à dire que les personnes âgées ne sont pas des êtres humains au même titre que les plus jeunes, et que leurs droits à des services de santé ne seraient donc pas considérés également. Une telle politique d'allocation va à l'encontre des principes de la justice et du respect des personnes âgées, car elle porte à leur endroit un jugement défavorable qui laisse croire ou qui suppose que ces dernières n'apportent rien à la société, qu'elles ont moins de mérite, qu'elles ne font aucun effort bénéficiant à la société et qu'elles ont donc moins de valeur.

Bien entendu, l'âge peut et doit être considéré, mais comme un facteur parmi d'autres dans l'entière particularité de la vie du patient âgé. Il faut choisir des traitements appropriés et efficaces, résister à la tentation de l'acharnement thérapeutique, et prendre en considération les désirs des malades. L'objectif de la médecine, qui est de guérir et de préserver la vie, ne peut pas toujours être atteint. Il arrive un moment où il faut rendre les armes et accepter l'issue «naturelle» de la mort. Les traitements médicaux doivent 
être utilisés non pas parce qu'ils sont disponibles, mais parce qu'ils servent à préserver ou prolonger la vie des patients, lorsque c'est dans leur intérêt et que cela améliore leur qualité de vie ou leur confort.

En définitive, l'âge à lui seul ne peut être un critère de répartition des ressources, car il ne permettra jamais de déterminer ce qui est dans l'intérêt des personnes âgées ni de juger de la qualité de leur vie. Il importe au plus haut point que les décisions ne soient pas prises selon des critères arbitraires, ou selon des considérations strictement politiques ou économiques.

\section{Conclusion}

"Les principes de respect, autonomie, lucidité, fidélité, humanité et justice sont des critères essentiels pour aborder les dilemmes éthiques des soins aux personnes âgées.»
Les principes de respect, autonomie, lucidité, fidélité, humanité et justice sont des critères essentiels pour aborder les dilemmes éthiques des soins aux personnes âgées. Toutefois, ils permettent ni de résoudre les problèmes mentionnés au début de cet article, ni de répondre à la question de savoir qui est responsable des soins aux gens âgés. C'est pourquoi nous concluons avec cet aspect des devoirs et des responsabilités.

En ce qui concerne les problèmes de compétence, de consentement éclairé et d'âgisme, les professionnels de la santé qui travaillent auprès des personnes âgées ont une lourde responsabilité, entre autres l'obligation morale de s'assurer que les personnes âgées compétentes ont l'occasion et la réelle liberté de faire des choix significatifs, à mesure qu'augmente leur dépendance envers les autres. Il faut respecter ces choix qui sont basés sur des valeurs personnelles et des projets de vie à découvrir. Il est alors essentiel que les professionnels de la santé n'imposent pas leurs valeurs personnelles et professionnelles, sous prétexte qu'ils savent mieux ce qui est dans l'intérêt du patient. Le respect des choix et des valeurs des malades oblige aussi les professionnels à ne pas les abandonner même s'ils ne partagent pas leurs valeurs. Il faut plutôt les référer à d'autres collègues plus sensibles à ces 
"L'équipe de soins de santé... doit considérer la personne âgée dans toute son intégrité..." valeurs. Il faut s'efforcer de trouver des moyens d'aider les patients compétents à surmonter les obstacles qui les empêchent de faire des choix sensés, et permettant aux patients incompétents d'exercer certains choix. L'équipe de soins de santé doit elle aussi agir dans le meilleur intérêt des malades, elle doit considérer la personne âgée dans toute son intégrité, et s'efforcer de répondre à l'ensemble de ses besoins physiques, psychologiques et sociaux. Ce n'est possible que si l'équipe fonctionne selon une approche interdisciplinaire.

En ce qui concerne l'établissement de soins de santé, l'une de ses responsabilités majeures est de constituer une communauté morale qui se charge de la discussion des questions éthiques, de la prévention et de la résolution des dilemmes éthiques. Il faut aussi établir des énoncés de mission, des politiques et des procédures qui insistent sur le respect de la dignité de toute personne, l'autonomie, la justice, l'égalité et l'équité. Il faut implanter dans le milieu une éthique de soins personnels et une organisation du système qui favorisent l'humanisation et la personnalisation des soins.

Quant au problème de l'allocation des ressources et de la responsabilité des soins aux personnes âgées, la situation demeure complexe. Étant donné les changements de modèle familial qu'a connus notre société, on peut prévoir qu'il y aura peu d'enfants pour prendre soin de leurs parents dans leur âge avancé. La plupart des gouvernements occidentaux ont versé des pensions et fourni des services pour que les citoyens âgés jouissent jusqu'à leur mort d'un niveau de vie acceptable et de services de santé adéquats. Ces programmes ont reçu une large part du budget national. Comme le pourcentage des contribuables diminue par rapport au nombre des gens âgés, il n'est pas sûr que la société dans son ensemble puisse continuer à financer ces programmes. Avec l'augmentation constante des coûts de la santé et de la dette nationale, il n'est pas certain que les soins médicaux que nous considérons actuellement comme normaux soient disponibles à l'avenir pour ceux qui seront alors âgés, et qui auront contribué pour une large part à en payer le coût. Nous faisons face à un problème dont la solution ne peut pas reposer uniquement sur 
"...les politiciens et les fonctionnaires... doivent s'assurer que les fonds consacrés aux soins de la santé, spécialement pour les personnes âgées, sont suffisants et équitables.»

«D'un point de vue préventif, nous pouvons acquérir des habitudes de vie et d'alimentation afin d'empêcher...que les maladies dites de modes de vie ne se répandent.» un groupe particulier. Il faut la collaboration et la concertation de tous. Ainsi les politiciens et les fonctionnaires qui dressent les budgets doivent s'assurer que les fonds consacrés aux soins de la santé, spécialement pour les personnes âgées, sont suffisants et équitables. Les institutions de santé, à cause de leur financement limité, doivent faire preuve de modération et d'équité, et doivent veiller à ce que tous les patients qui en ont besoin aient le même accès aux services selon un système équitable de rationnement (d'ordinaire une liste d'attente prenant en considération la gravité du besoin médical). Parmi les professionnels de la santé, les médecins ont une responsabilité particulière dans le rationnement des ressources. En effet, ils sont responsables d'environ $80 \%$ des coûts des soins de santé. Ils se doivent de ménager les ressources rares, d'éviter de soumettre les patients à des examens coûteux, et de prescrire des médicaments dont le bénéfice est douteux.

Enfin, les usagers des services de santé ont eux aussi une responsabilité dans le rationnement des ressources de santé. Nous nous sommes faits à l'idée qu'un programme universel d'assurance maladie nous donne accès sans restriction aux hôpitaux et aux médecins, sans que cela nous coûte quoi que ce soit. Il est évident que nous avons un prix à payer comme membres de la société, et que nous pouvons apporter notre contribution à une utilisation plus rationnelle des soins de santé. Ainsi, nous pouvons éviter les exigences excessives qui expliquent l'abus des services de santé, par exemple, la fréquentation inappropriée des services d'urgence des hôpitaux, la consultation de nombreux spécialistes pour le même problème médical, ou l'insistance de la famille pour traiter agressivement des parents en phase terminale, etc. D'un point de vue préventif, nous pouvons acquérir des habitudes de vie et d'alimentation afin d'empêcher, autant que possible, que les maladies dites de modes de vie ne se répandent.

En ce sens, les personnes âgées ont aussi un rôle à jouer. Elles devraient être encouragées à demander l'aide, quand elles en ont besoin, des membres de leur famille, des groupes communautaires, des institutions de soins médicaux et du gouvernement. Tout en développant l'autonomie des personnes âgées, nous devons aussi promouvoir l'interdépendance, en construisant des résidences 
"... il faut susciter la prise en charge personnelle et la solidarité de chaque membre de la société...» adaptées à leurs besoins et en mettant sur pied, des programmes de visites à domicile, de transport en commun ou de co-voiturage, des cuisines communautaires, etc. Les personnes âgées doivent apprendre à s'entraider et à collaborer avec les autres groupes d'âge.

Notre société occidentale insiste à bon droit sur les droits et privilèges. Il ne faudrait pas oublier leur corollaire, à savoir les devoirs et les responsabilités. Plus que jamais, il faut susciter la prise en charge personnelle et la solidarité de chaque membre de la société, de la naissance à la mort.

À toutes et tous s'ouvre le vaste chantier d'une éducation continue et permanente.

\section{Notes}

1. Callahan (1987) élabore davantage cette position dans son ouvrage Setting Limits : Medical Goals in an Aging Society.

2. Saint-Arnaud (1993) définit l'aptitude comme la capacité de comprendre les informations qui sont pertinentes en regard de la décision à prendre, de délibérer en fonction de ses valeurs et de ses buts, et enfin la capacité de communiquer ses décisions (p. 35).

3. Comme le note Harvey (1993), la doctrine juridique qui soustend le consentement éclairé repose sur le droit fondamental de l'individu de déterminer ce qu'il adviendra de sa personne.

\section{Bibliographie}

ASSOCIATION MÉDICALE CANADIENNE (1987). Les soins de santé pour gens âgées: les défis d'aujourd'hui, des solutions pour demain, Ottawa, AMC.

BATTIN, Margaret Pabst (1987). «Choosing the Time to Die :The Ethics and Economics of Suicide in Old Age», dans Stuart Spicker et al., eds. Ethical Dimensions of Geriatric Care:Value Conflicts for the 21st Century, Dordrecht/Boston, D. Reidel, 161-189.

BURGALASSI, S. (1977). "La condition de la vieillesse: une approche globale à niveau anthropologique et sociologique», Médecine et morale, 263-264.

CALLAHAN, Daniel (1987). «Terminating Treatment : Age as a Standard», Hastings Center Report, vol. 17 , no $5,21-25$. 
CRANLEY GLASS, Karen (1993). «Éthique et rationnement des soins de santé en fonction de l'âge du patient", dans Conseil consultatif national sur le troisième âge, Écrits en Gérontologie (13) - Questions éthiques et vieillissement, Ottawa, ACCNTA, 71-86.

DANIELS, Norman (1987). «Equal Opportunity,Justice, and Health Care for the Elderly:A Prudential Account», dans Stuart Spicker et al., eds. Ethical Dimensions of Geriatric Care:Value Conflicts for the 21st Century, Dordrecht/Boston, D. Reidel, 197-221.

DANIELS, Norman (1988). Am I My Parents' Keeper? An Essay on Justice Between the Young and the Old, New York, Oxford University Press.

FRIED, Charles (1974). Medical Experimentation. Personal Integrity and Social Policy, Amsterdam, Oxford, North-Holland Publishing Company.

HARVEY, William R.C. (1993). «Éthique et soins de santé des ainé-e-s», dans Conseil consultatif national sur le troisième âge, Écrits en Gérontologie (13) - Questions éthiques et vieillissement, Ottawa, CCNTA, 51-69.

KENYON, Gary M. et Warren DAVIDSON (1993). «L'éthique dans une société vieillissante», dans Conseil consultatif national sur le troisième âge, Écrits en Gérontologie (13) - Questions éthiques et vieillissement, Ottawa, CCNTA, 19-32.

LÉGARÉ, Jacques (1996). «Le vieillisssement des populations», Interface, vol. 17, no 3, 28-35.

ROY, David J., John R. WILLIAMS, Bernard M. DICKENS et Jean-Louis BAUDOUIN (1995). La bioéthique. Ses fondements et ses controverses, Saint-Laurent (Québec), Éditions du Renouveau pédagogique inc.

ROYAL COLLEGE OF PHYSICIANS AND SURGEONS OF CANADA (Collège Royal des médecins et chirurgiens du Canada) (1988). «Informed Consent: Ethical Considerations for Physicians and Surgeons", Annals of The Royal College of Physicians and Surgeons of Canada, 21/2, (encarté).

SAINT-ARNAUD, Jocelyne (1993). «Autonomie, autodétermination et processus décisionnel quant aux traitements de fin de vie», dans Conseil consultatif national sur le troisième âge, Écrits en Gérontologie (13) - Questions éthiques et vieillissement, Ottawa, CCNTA, 33-50.

SIMARD, Noël (1984). La conscience morale et sa dimension chrétienne dans une vision personnaliste, (Thèse de doctorat), Rome, Université pontificale grégorienne.

STATISTIQUES CANADA (1991). Annuaire du Canada 1992 / $125^{e}$ Anniversaire, Octobre 1991, Ottawa, Statistiques Canada - Division des publications.

TETTAMANZI, Dionigi (1989). "Aspects éthiques du troisième âge», Dolentium Hominum, no 10, (Année IV - N. 1), 80-86.

THOMASMA, David C. (1984). «Freedom, Dependency, and the Care of the Very Old», Journal of the American Geriatics Society, 32/12, 906-914,

VERSPIEREN, Patrick (1984). Face à celui qui meurt, Paris, Desclée de Brouwer. 